\title{
Automated Image Identification Method for Flood Disaster Monitoring In Riverine Environments: a Case Study in Taiwan
}

\author{
Jyh-Horng Wu, Chien-Hao Tseng, Lun-Chi Chen, Shi-Wei Lo, Fang-Pang Lin \\ National Center for High-Performance Computing, National Applied Research Laboratories, Taiwan
}

\begin{abstract}
As the globe undergoes extreme climate changes, disaster events and their disaster scale continue to increase; therefore, it has become imperative to devise disaster prevention measures. Traditional flood monitoring devices, while operating in harsh environments, are often influenced by changes in weather conditions such light, rain, and fog. Consequently, recorded images are often blurred or damaged, which increases the possibility of errors in judgment or delays in the hazard mitigation process. In this study, an automated identification method for flood monitoring based on real-time video images is proposed. The method can be used by the Water Conservancy Agency of the Ministry of Economic Affairs automated for automated identification of flooded areas and for automated determination of the water levels of the main rivers from image data. This paper presents results to show that the capability to detect temporal changes in image sequences is crucial for an automated image-based flood alarm system used in disaster-monitoring applications.
\end{abstract}

Keywords-flood alarm system; flood monitoring; hazard mitigation; image identification.

\section{INTRODUCTION}

In recent years, flooding has occurred in numerous regions worldwide. The main causes of flooding are heavy rain, hurricanes, and tsunami, among which heavy rain is the dominant factor. Rain that falls for extended periods causes rivers to overflow, flooding the surrounding areas. In September 2008, Typhoon Sinlaku, which passed over the Philippines, Taiwan, China, and Japan, caused enormous damage through heavy rains, flooding, and landslides. In Taiwan, the accumulated rainfall reached a new record of $1000 \mathrm{~mm}$. The hurricanes caused at least \$22.2 million in damages to the agriculture sector, and 14 people were killed during the typhoon [1]. Because natural disasters cannot be prevented, researchers have focused on using current technology to reduce the resulting losses and effects. Various methods can be used to monitor and prevent flooding events, and video surveillance systems have been widely and effectively used in recent years for monitoring flooding events.

Flood monitoring at embankments, bridges, levees, and dams is essential for flood prevention. One of the most challenging problems in flood response is the precise localization of flood risk; this task is performed by early warning system (EWS) for flood prevention and disaster management. EWS are extensively used for mitigating flood risk, detecting abnormalities, and predicting the onset of flooding, and they involve the use of remote sensors [2, 3]. Traditionally, EWS have been used for monitoring flooding by employing remote sensing technology such as satellite imaging and electronic sensors installed near rivers and seaports.. Image analysis technology has been widely applied in numerous critical fields, such as medicine and aerospace. More recently, the technology has been determined to be useful in environmental object analysis [4]. A decision-theoretic methodology that is suitable for modeling and evaluating forecast response systems can be used to develop automated detection systems that can issue flood warnings $[5,6]$. To provide real-time flood condition information and timely flood warnings to authorities, web-based decision support systems are typically employed for flood management.

To resolve challenges faced in flood monitoring and warning systems, flood-monitoring techniques were combined to realize an image-based flood alarm module (IFAM). The IFAM served as the foundation for a morphological image processing system that can be used to detect and identify flood conditions from real-time video images [7]. In this paper, an image-based flood alarm system that is capable of automated flood detection and identification is proposed; the flood alarm system is based on an image-processing model and mathematical morphology methods. An image analysis methodology for identifying mainstream rivers can be combined with the mean-shift (MS) and region growing (RegGro) algorithms $[8,9]$. Experimental results were evaluated using various data sets, and it was observed that the proposed alarm system can effectively identify flood hazard areas from image sequences.

The remainder of this paper is organized as follows. Section II presents a preliminary background for the application of the image-based flood alarm system to an image processing module. Section III discusses the descriptions and selection of field measurements. Section IV presents the results and discussion of the designed experiments. Finally, the conclusion is provided in Section $\mathrm{V}$.

\section{MATERIALS AND METHODOLOGIES}

\section{A. Image-Based Flood Alarm Framework}

The image-based flood alarm (IFA) is an image-based flood alert system that facilitates proactive monitoring, assessment, and early response to rising water levels and associated inundation in real time. The IFAM receives video streams from digital camera sensors installed 
around rivers. The video streams are decomposed into JPEG images and filtered for image enhancement. The module can then calculate water levels and assess the accompanying flood risk. Fig. 1 shows the proposed flood alert monitoring system. The processing methods involved in the detection and identification of the flooded areas are as follows.

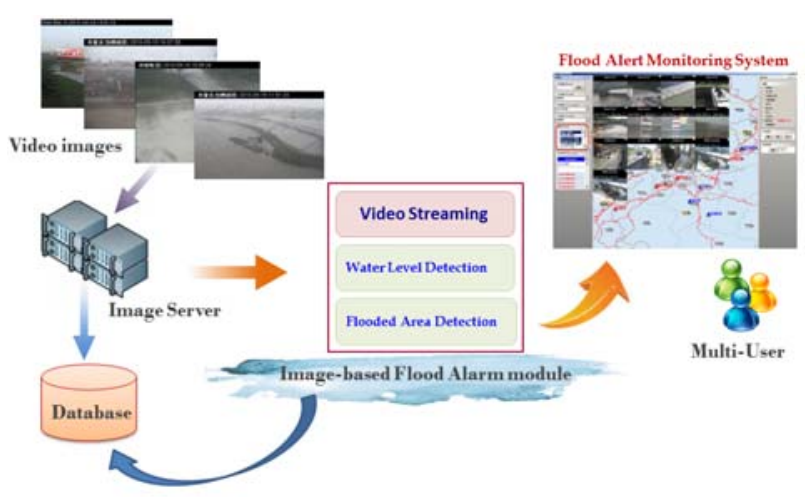

Figure 1. Sketch of the proposed flood-alert monitoring system.

B. Identifying Flooded Areas by Using the Mean-Shift and Region Growing Algorithms

The MS algorithm is a powerful nonparametric clustering algorithm based on the nonparametric estimation of probability density functions, and it can be used to estimate local density gradients of similar pixels. These gradient estimates are used in an iterative procedure to determine the peaks in the local density. In the iterative stage of the MS procedure, each pixel is assigned an MS point $M\left(x_{i}\right)$, which is initialized to coincide with the pixel. The multivariate kernel density estimator with a bandwidth matrix $\mathbf{H}$ and computed at point $x$ is calculated as follows:

$$
\hat{f}(x)=\frac{1}{n} \sum_{i=1}^{n} K_{H}\left(x-x_{i}\right)
$$

where the $n$ data points $x_{i}$ represent a sample with an unknown density $f$, or in the case of images or videos, the pixels themselves.

$$
K_{H}(x)=|\mathbf{H}|^{-1 / 2} K\left(\mathbf{H}^{-1 / 2} x\right)
$$

where $K(x)$ is a $d$-dimensional kernel function satisfying the conditions for asymptotic unbiasedness, consistency, and the uniform consistency of the gradient of the density estimate. The term $\mathbf{H}$ is a symmetric positive definite $d \times d$ bandwidth matrix.

If a normal (Gaussian) kernel is used, the MS vector becomes

$$
m_{h, K}(x)=\frac{\sum_{i=1}^{n} x_{i} \exp \left(\left\|\frac{x-x_{i}}{h}\right\|^{2}\right)}{\sum_{i=1}^{n} \exp \left(\left\|\frac{x-x_{i}}{h}\right\|^{2}\right)}-x .
$$

The term $m_{h, K}(x)$ is proportional to the normalized density gradient and always points in the direction of maximal density increment.

The MS algorithm is an iterative procedure involving the following steps:

Step 1: Compute the MS vector for point $m_{h, K}\left(x_{i}^{k}\right)$ according to (3).

Step 2: Update the current position: $x_{i}^{k+1}=x_{i}^{k}+m_{h, K}\left(x_{i}^{k}\right)$

Step 3: Repeat Steps 1 and 2 until convergence; in other words, until the gradient of density functions at the point is zero (i.e., $\nabla \hat{f}_{h, K}(x)=0$ ).

The RegGro algorithm is a simple region-based image segmentation algorithm. The purpose of using RegGro is to group pixels into meaningful regions, starting from a specific seed pixel and spreading to neighbors that satisfy the growing rule. The fundamental disadvantage of intensity-based region segmentation is that intensity provides no spatial information and the settled threshold is a single value or a set of gray levels. Hence, RegGro uses a compact dynamic mean intensity with a settled threshold (intensity distance) to implement the growing rule. The RegGro rule pseudocode can be presented as follows:

RegGro rule pseudocode
//For each pixel p
for all p in the image
//Copy the previous state of p and the mean intensity of all
q
labels_new = labels; Mean_Intensity(q)
//All neighbors q try to spread to the current cell p
for all neighbors q
if(Intensity(p) < Mean_Intensity(q))
$\quad / / G r o w i n g$ successful spread out to the current
cell p, growing.
labels_new(p) = labels(q)
end if
end for
end for

\section{DESCRIPTION OF CASE STUDY}

In this study, the proposed IFAM was employed to identify flooded areas. All the sample images of flooded areas captured from streaming video were evaluated. All images were in JPEG format, exhibiting a 24-bit color depth and a spatial resolution of $352 \times 240$. Field experiments for the measurement were conducted on the Nan-Hu No. 2 Bridge and Ma-Ling-Keng River in Taiwan. 
The Nan-Hu No. 2 Bridge data were recorded during Typhoon Sinlaku, which induced a rainstorm on September 14, 2008. The video images were captured between noon (when the rain began) and nightfall (flood tiding).
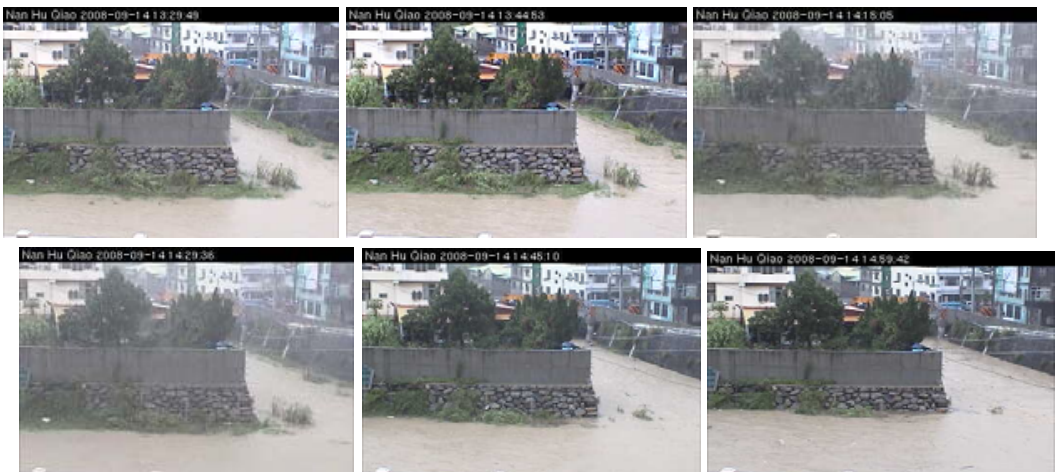

Figure 2. Sample image sequences for the case of the Nan-Hu No. 2 Bridge dataset (from top to bottom, left to right)
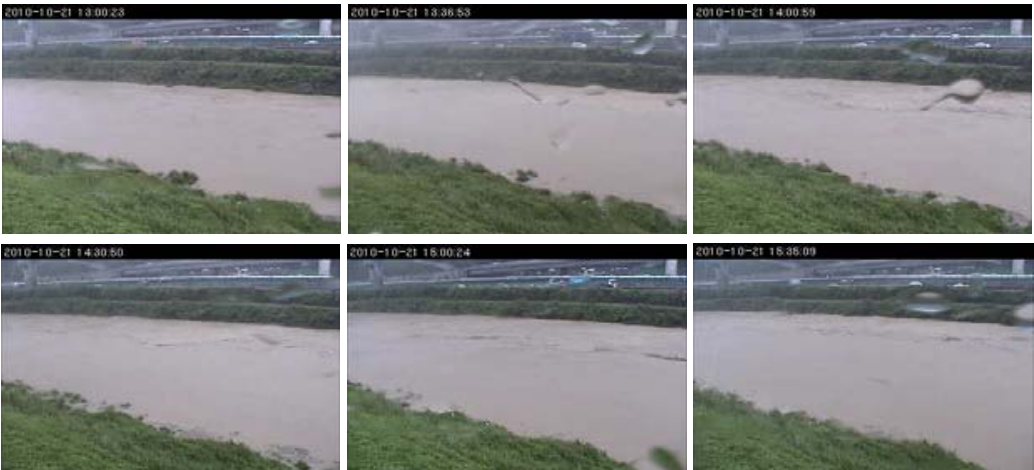

Figure 3. Image sample sequences for the case of Ma-Ling-Keng River (from top to bottom, left to right).

\section{RESULTS AND DisCUSSION}

In this paper, the proposed IFAM was used to evaluate test areas in various image sets for selecting the optimal image segmentation and flood identification methods. The automated identification method for conducting flood monitoring is based on real-time video images. Figs. 4 and 5 show example images from the two datasets, obtained using the proposed algorithm.

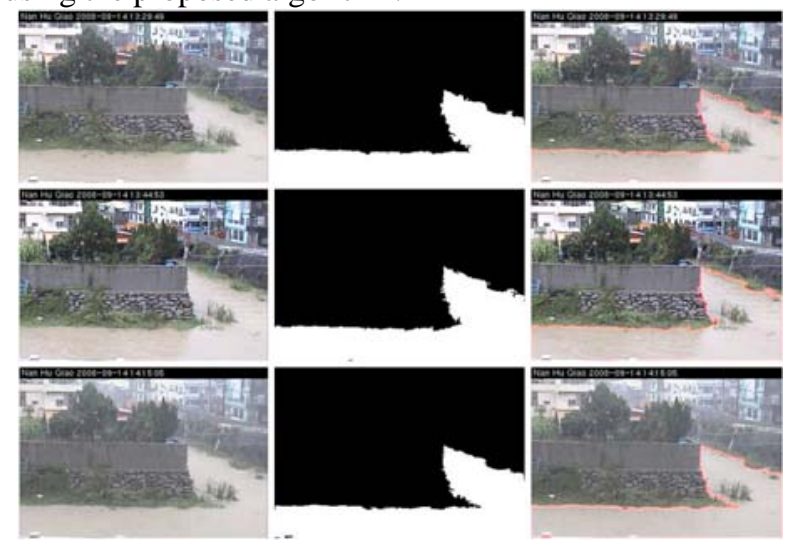

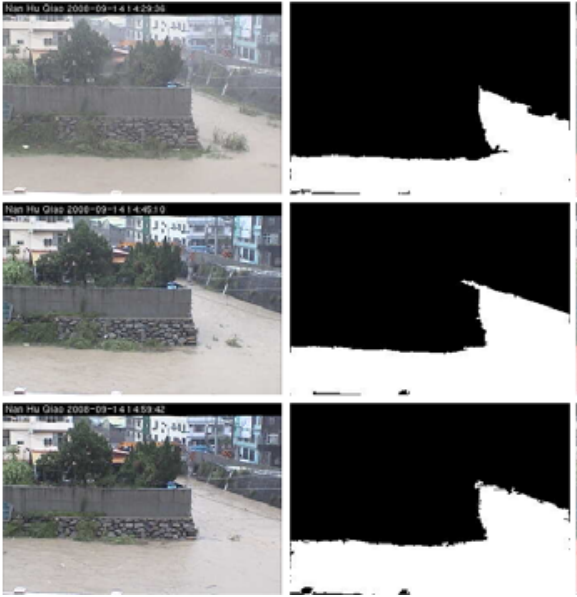

(a) (b)

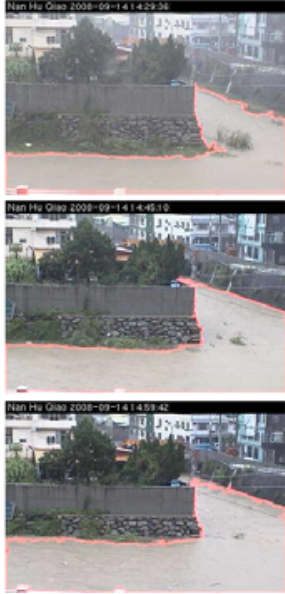

(c)
Figure 4. Nan-Hu No. 2 Bridge image set. From left to right: (a) Input image, (b) segmented image, and (c) identified image. 


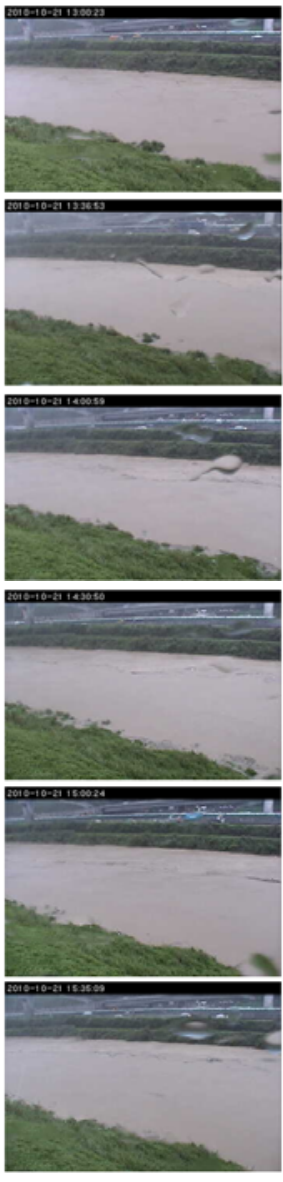

(a)
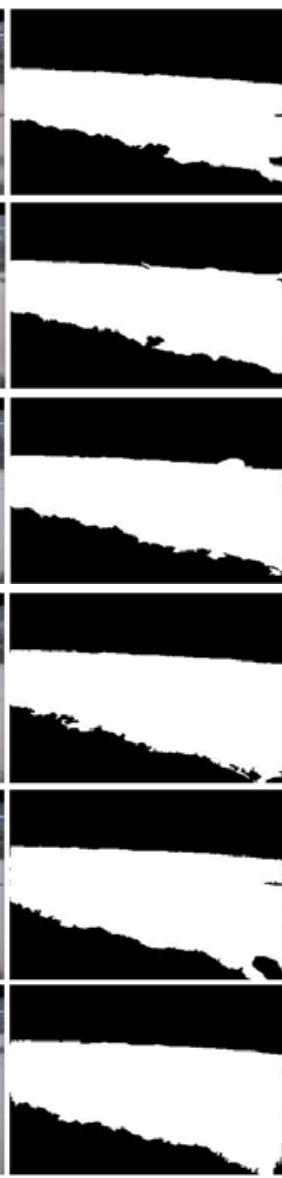

(b)
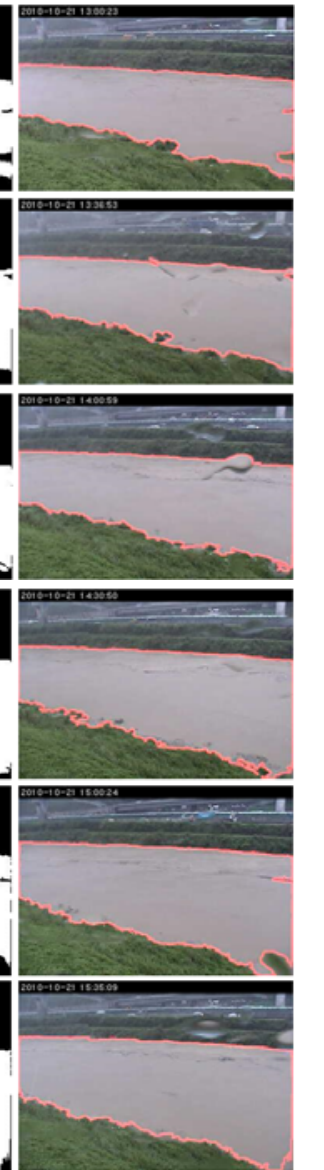

(c)
Figure 5. Ma-Ling-Keng River image set. From left to right: (a) Input image, (b) segmented image, and (c) identified image.

The first step in image processing is converting the color space from the RGB domain to the HSV domain. The HSV color space is preferred because it is more perceptually relevant than the Cartesian coordinates represented by the RGB domain. To distinguish a river object from its background, histogram equalization was used to enhance the object contrast. The MS methodology was then used to classify video images into building, plant, river, and embankment. Finally, the RegGro algorithm was used for segmentation for forming a binary mask. The outlines of the river areas were preserved within the binary mask.

In this paper, an image-based flood alarm system was proposed to achieve automated flood detection and identification based on the image-processing model and mathematical morphology methods. The image analysis methodology can be combined with MS and RegGro algorithms. Experiments conducted in the present study indicated that the IFAM receives video streams from digital camera sensors, continually calculates image data, and automatically detects flooded areas. The automated flood-alert monitoring system presents results concerning flooded areas; the system can provide real-time water resource information obtained from all monitoring stations to multiple users.

\section{V.CONCLUSIONS}

This paper presents an automated flood-alert monitoring system for monitoring river conditions from real-time video images; the monitoring system detects and identifies flooded areas. The mathematical morphology method was used to identify river areas. The approach is designed to improve the identification accuracy in adverse weather conditions while preserving accuracy in clear weather conditions. Crucial attributes that should be considered for achieving high identification performance are fog and rain, which strongly affect morphological features. The experimental results demonstrated the suitability of the proposed automatic image-based flood alarm system, which can effectively identify flood hazard areas from image sequences.

\section{REFERENCES}

[1] Hurricane Season 2008: Typhoon Sinlaku (Western Pacific Ocean): Sinlaku Was a Killer in Taiwan, NASA Official, http://www.nasa.gov/

[2] Basha, E.A., Ravela, S. \& Rus, D., Model-Based Monitoring for Early Warning Flood Detection. 6th ACM Conference on Embedded Network Sensor Systems, pp. 295-308, 2008.

[3] Krzhizhanovskaya, V.V., Shirshov, G.S., Melnikova, N.B., Belleman, R.G., Rusadi, F.I., Broekhuijsen, B.J., Gouldby, B.P., Lhomme, J., Balis, B., Bubak, M., Pyayt, A.L., Mokhov, I.I., Ozhigin, A.V., Lang, B. \& Meijer, R.J., Flood early warning system: design, implementation and computational modules. Procedia Computer Science, 4, pp. 106-115, 2011.

[4] Blaschke, T., Object based image analysis for remote sensing. ISPRS Journal of Photogrammetry and Remote Sensing, 65(1), pp. 2-16, 2010.

[5] Krzysztofowicz, R. \& Davis, D.R., A Methodology for Evaluation of Flood Forecast-Response Systems. Water Resources Research, 19(6), pp. 1423-1429, 1983.

[6] Albanese, A., Disabato, F., Vigna, R. \& Terzo, O., Early warning system for flood events and automatic classification of low resolution satellite imagery: ITHACA approach. International Workshop on Remote Sensing for Disaster Applications, pp. 1-6, 2008.

[7] Sunkpho, J. \& Ootamakorn, C., Real-time flood monitoring and warning system. Songklanakarin Journal of Science Technology, 33(2), pp. 227-235, 2011.

[8] Cheng, Y., Mean Shift, Mode Seeking, and Clustering. IEEE Transactions on Pattern Analysis and Machine Intelligence, 17(8), pp. 790-799, 1995.

[9] Adams, R. \& Bischof, L., Seeded Region Growing. IEEE Transactions on Pattern Analysis and Machine Intelligence, 16(6), pp. 641-647, 1994. 